\title{
Morfometria do oviduto de poedeiras comerciais semipesadas submetidas a diferentes métodos de muda forçada
}

\author{
Morphometry of the oviduct of the brown egg layer hens submitted different methods of molt induction
}

\author{
Cristiane Soares da Silva Araújo ${ }^{\mathrm{I}}$ Silvana Martinez Baraldi ArtoniI ${ }^{\mathrm{II}}$ Lúcio Francelino Araújo ${ }^{\mathrm{III}}$ \\ Otto Mack Junqueira ${ }^{\mathrm{IV}}$ Luis Carlos Garibaldi Simon BarbosaV César Gonçalves de LimaVI
}

RESUMO

Este experimento foi conduzido com o objetivo de avaliar a morfometria do oviduto de poedeiras submetidas a diferentes métodos de indução de muda forçada. Duzentas e oito aves com 58 semanas de idade foram distribuídas em um delineamento inteiramente casualizado, com quatro tratamentos (método Califórnia, dieta com alto teor de zinco, dieta com baixo cálcio e com baixo sódio) e quatro repetições de 12 aves cada. Avaliou-se a biometria do oviduto no início do experimento, no 28ㅇ. dia e em três períodos subseqüentes à realização da muda forçada, analisando-se o peso corporal da ave, a percentagem do oviduto e do ovário, o número de pregas do magno e do istmo e o comprimento das diferentes partes que compõem o oviduto. No período pós-muda, as características macroscópicas do oviduto não sofreram influência dos tratamentos estudados.

Palavras-chave: manejo nutricional, pós-muda, sistema reprodutor.

\section{ABSTRACT}

This experiment was aimed at evaluating the performance, oviduct morphometric, hormone concentration and bone density in laying hens submitted of the different methods of molt induction. Two hundred-eight laying hens, 58 weeks of age, were randomly divided into four treatments groups (california method, diet of high-zinc, diet of low-calcium and diet of low-sodium) and four replicates. The experiment evaluated biometrics characteristics on day 1, 28 of the experiment and in three periods postmolt: body weight, ovary percentage, oviduct percentage, number of folds of the magnum and isthmus, and length of the different parts of oviduct. In the postmolt period, the morphometric analysis of the birds were not affected by any of the experimental treatments.

Key words: nutricional manage, postmolt, reproductive system.

\section{INTRODUÇÃO}

A muda de penas é um processo natural de todas as aves. As aves silvestres fazem muda de penas anualmente. Nestas aves, a produção de ovos é pequena e a muda está associada ao ciclo reprodutivo. Contudo, o processo de muda vai além da substituição de penas. Estudos revelam que um significativo incremento da taxa de metabolismo, aumento da síntese de algumas proteínas, osteoporose, perda de gordura e supressão do sistema imune ocorrem durante esse evento cíclico anual da ave (KUENZEL, 2003).

As poedeiras comerciais são hoje selecionadas visando-se a uma alta produção de ovos e não iniciam o processo de muda antes de completarem um longo e intenso período de postura. O período de muda, em condições naturais, concretiza-se em cerca de quatro meses. Contudo, é possível provocar artificialmente a muda de penas e concretizá-la em oito semanas ou menos, dependendo do programa que se venha a adotar.

\footnotetext{
'Departamento de Zootecnia, Faculdade de Ciências Agrárias e Veterinárias, Universidade Estadual Paulista (FCAV, UNESP), Jaboticabal, SP, Brasil.

"Departamento de Morfologia e Fisiologia Animal, FCAV, UNESP, Jaboticabal, SP, Brasil.

IIIDepartamento de Zootecnia, Faculdade de Zootecnia e Engenharia de Alimentos, Universidade de São Paulo (FZEA, USP). Av.

Duque de Caxias Norte, 225, 13635-900, Pirassununga, SP, Brasil. E-mail:lfaraujo@usp.br.

${ }^{\text {IV }}$ Departamento de Zootecnia, FCAV, UNESP, Jaboticabal, SP, Brasil.

`Departamento de Apoio, Produção e Saúde Animal, UNESP, Araçatuba, SP, Brasil.

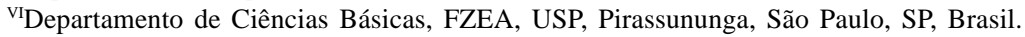


No que se refere à idade de realização da muda, deve-se levar em consideração a qualidade e o preço dos ovos. Se a qualidade é boa e os ovos têm atingido um bom preço de mercado, realiza-se a muda numa idade que tem sido empregada normalmente no setor avícola (aproximadamente 70 semanas). Por outro lado, se a qualidade é ruim e os preços são baixos, uma antecipação da muda pode ser justificada. Nos Estados Unidos, 90\% dos lotes sofrem muda entre 60 e 80 semanas de idade (BELL, 2003).

Vários métodos têm sido utilizados para realizar a muda. Esses métodos incluem a restrição alimentar de 10 dias (CHRISTMAS et al., 1985), jejum hídrico de dois dias (NORTH \& BELL, 1990), dieta com baixo nível de cálcio (BREEDING et al., 1992) ou baixo nível de sódio (BERRY \& BRAKE, 1985) e dietas com altos níveis de zinco (BRAKE, 1993). Cada método pode ser usado sozinho ou em combinação com os outros métodos. Todos os métodos necessitam que haja perda do peso corporal e que cesse a postura (SHIPPEE et al., 1979). Embora a retirada da ração seja considerada um método fácil de aplicação da muda e os resultados sejam os melhores, têm sido questionados aspectos relacionados com o bem estar da ave (WEBSTER, 2003).

A temperatura corporal no período de muda forçada, durante o jejum, sofre um decréscimo, mas volta a subir quando o alimento é reintroduzido, sendo este fato coincidente com a perda de pena pelas poedeiras (BRAKE \& THAXTON, 1979a). A indução da muda forçada, através do método do jejum, induz perda de peso nas poedeiras ao redor de $25 \%$, sendo que um quarto deste efeito é atribuído diretamente à diminuição do peso do fígado, do ovário e do oviduto. (BRAKE \& THAXTON, 1979b).

A taxa de produção (\% de postura) pré-muda é um importante fator que interfere nas alterações fisiológicas observadas durante a muda forçada. Assim, poedeiras com percentagem de postura menor, antes da muda, apresentam mudanças psicológicas mais significativas que aves com maior índice de produção, tais como, redução no peso do ovário, do oviduto, no peso corporal e de gordura, e aumento da concentração de colesterol e proteína no plasma, do que aves com maior índice de produção no plasma (ROLAND \& BRAKE, 1982).

Baseado no fato de que o processo da muda forçada envolve aspectos relacionados a alterações macroscópicas ocorridas no oviduto e de que existe uma grande preocupação em se utilizar métodos alternativos, menos agressivos e com resultados satisfatórios para se induzir este processo, é que se objetivou avaliar os efeitos dos diferentes métodos de indução da muda forçada, em poedeiras comerciais semipesadas, sobre a biometria do trato reprodutivo.

\section{MATERIAL E MÉTODOS}

O experimento foi realizado no aviário da Faculdade de Ciências Agrárias e Veterinárias do Campus de Jaboticabal - UNESP. O galpão de postura utilizado foi do tipo convencional, com gaiolas de arame galvanizado com quatro compartimentos, distribuídas lateralmente em dois andares. Trabalhou-se com duas aves por gaiola e o comedouro utlizado foi de madeira, o qual percorria toda a extensão frontal das gaiolas, e o bebedouro foi tipo copo plástico. Durante o período de indução da muda forçada, as aves receberam somente a luz natural. Após esse período, reintroduziu-se a luz artificial progressivamente até que fossem atingidos 17 horas de luz/dia.

Foram utilizadas 208 aves da linhagem Isa Hissex Brown, com 58 semanas de idade, distribuídas em 16 parcelas de 13 aves cada. No início do experimento, as aves foram submetidas a seleção, pesadas e distribuídas aleatoriamente nas parcelas experimentais. Os tratamentos experimentais estudados são descritos na tabela 1 .

As dietas foram formuladas à base de milho, farelo de soja e farelo de trigo, seguindo-se as recomendações do NRC (1994) (Tabela 2). A dieta de postura fornecida pós-muda seguiu a recomendação do manual de manejo da linhagem.

As medidas biométricas do oviduto ocorreram no início da fase experimental e as demais

Tabela 1 - Descrição dos tratamentos experimentais.

\begin{tabular}{llc}
\hline Tratamento & \multicolumn{1}{c}{ Período de indução da muda } & Período de descanso * \\
Califórnia ou restrição & Restrição alimentar até $10^{\circ}$. dia & $11^{\circ}$. ao $28^{\circ}$. dia \\
\hline Dieta alto nível de zinco & $20.000 \mathrm{ppm}$ óxido de zinco até o $10^{\circ}$. dia & $11^{\circ}$. ao $28^{\circ}$. dia \\
Dieta baixo nível de cálcio & $0,1 \%$ de cálcio até o $14^{\circ}$. dia & $15^{\circ}$. ao $28^{\circ}$. dia \\
Dieta baixo nível de sódio & $0,05 \%$ de sódio até o $14^{\circ}$. dia & $15^{\circ}$. ao $28^{\circ}$. dia \\
\hline
\end{tabular}

*Fornecimento de milho moído e suplemento vitamínico e micromineral para frangas.

Ciência Rural, v.37, n.1, jan-fev, 2007. 
Tabela 2 - Composição percentual das dietas experimentais.

\begin{tabular}{|c|c|c|c|c|}
\hline Ingrediente & Alto $\mathrm{Zn}$ & Baixo Ca & Baixo Na & Postura \\
\hline Milho & 69,64 & 69,64 & 69,64 & 65,41 \\
\hline Farelo de soja & 14,67 & 14,67 & 14,67 & 18,87 \\
\hline Farelo de trigo & 10,00 & 10,00 & 10,00 & 4,50 \\
\hline Lisina $\mathrm{HCl}, 99 \%$ & 0,00 & 0,00 & 0,00 & 0,02 \\
\hline Calcário calcítico & 1,31 & 0,00 & 1,31 & 9,02 \\
\hline Fosfato bicálcico & 1,00 & 0,15 & 1,00 & 1,26 \\
\hline Sal comum & 0,28 & 0,28 & 0,04 & 0,42 \\
\hline Supl. Vit/Mineral* & 0,50 & 0,50 & 0,50 & 0,50 \\
\hline Óxido de zinco & 2,00 & 0,00 & 0,00 & 0,00 \\
\hline Inerte & 0,60 & 4,76 & 2,84 & 0,00 \\
\hline Total & 100 & 100 & 100 & 100 \\
\hline \multicolumn{5}{|c|}{ Níveis nutricionais } \\
\hline Energia metabolizável, kcal/kg & 2.900 & 2.900 & 2.900 & 2.750 \\
\hline Proteína bruta (\%) & 14,25 & 14,25 & 14,25 & 15,00 \\
\hline Cálcio (\%) & 0,80 & 0,10 & 0,80 & 3,80 \\
\hline Fósforo disponível (\%) & 0,30 & 0,30 & 0,30 & 0,34 \\
\hline Metionina (\%) & 0,23 & 0,23 & 0,23 & 0,40 \\
\hline Metionina + cistina (\%) & 0,48 & 0,48 & 0,48 & 0,65 \\
\hline Lisina (\%) & 0,62 & 0,62 & 0,62 & 0,72 \\
\hline Sódio (\%) & 0,15 & 0,15 & 0,05 & 0,20 \\
\hline Ácido liniléico (\%) & 1,50 & 1,50 & 1,50 & 1,35 \\
\hline
\end{tabular}

*Níveis de garantia do suplemento vitamínico e mineral por quilograma do produto: vit. A (UI):176.000, vit. $\mathrm{D}_{3}$ (UI):40.000, vit. E (mg): 500, vit. K3 (mg): 100, vit. B $(\mathrm{mg})$ : 36, vit. $\mathrm{B}_{2}$ (mg): 200, vit. $\mathrm{B}_{6}(\mathrm{mg}): 50$,:vit. $\mathrm{B}_{12}(\mathrm{mcg})$ : 560, biotina(mg): 3, pantotenato (mg): 500 , niacina (mg): 700, ac. fólico (mg): 30, promotor crescimento (g): 2, colina (mg): 20, cobre (mg): 300, iodo (mg): 24, selênio(mg): 3, manganês (mg): 1.800, zinco (mg): 1.200, ferro (mg): 1.100, metionina (g): 20, promotor de crescimento (g): 10, coccidicidiostático (g): 10 antifúngico (mg): 200, B.H.T. (g): 1 e veículo q. s. p. (g): 1.000.

avaliações se deram no $28^{\circ}$ dia do experimento e nos três períodos subseqüentes. Para tanto, quatro aves de cada tratamento foram sacrificadas, em cada período, para a avaliação do peso corporal da ave, do peso relativo do ovário, do peso relativo do oviduto, do número de pregas do magno e do istmo e do comprimento das diferentes partes do oviduto: infundíbulo, magno, istmo, glândula da casca e vagina. Ao coletar o oviduto, este foi estendido em uma bancada provida de fita métrica para realização das medidas de comprimento dos diferentes compartimentos.

Os dados obtidos foram analisados usandose o procedimento ANOVA SAS (1996) e, quando necessário, o teste de comparação de médias utilizado foi o de Tukey a $5 \%$.

\section{RESULTADOS E DISCUSSÃO}

São apresentados, na tabela 3, os resultados referentes à avaliação, macroscópica do ovário e do oviduto antes da muda forçada. Observou-se que não houve diferença significativa das características avaliadas, a saber, percentagem do ovário e do oviduto, comprimento dos compartimentos do oviduto e número de pregas do magno e do istmo $(\mathrm{P}>0,05)$. Esses resultados eram esperados, já que o processo de indução da muda forçada ainda não havia iniciado.

Levando-se em consideração que os trabalhos com muda forçada encontrados na literatura apresentam somente uma avaliação do oviduto em termos de peso relativo, um estudo mais apurado com a verificação do comprimento dos diferentes compartimentos do oviduto, bem como a contagem do número de pregas do magno e do ístmo, foram realizados. Entretanto, a análise de variância não mostrou diferenças significativas para peso relativo do ovário e do oviduto, bem como do comprimento das diferentes partes do oviduto e do número de pregas do magno e do istmo durante o período pós-muda $(\mathrm{P}>0,05)$ (Tabelas 3, 4 e 5).

O infundíbulo consiste de uma estrutura tubular de 4 a 10cm, de parede fina, com região cônica, seguindo-se por outra tubular com pregas em espiral suave. Também chamada de glândula albuminífera, possui estrutura tubular, de parede mais espessa, com 20 a 48cm de comprimento (é a parte mais longa), rico em glândulas tubulares dentro das pregas longitudinais 
Tabela 3 - Peso relativo do ovário e do oviduto, comprimento dos diferentes compartimentos do oviduto e número de pregas do magno e do istmo de poedeiras comerciais antes do início da muda forçada.

\begin{tabular}{|c|c|c|c|c|c|}
\hline Peso relativo (\%) & Restrição & Zinco & Cálcio & Sódio & CV (\%) \\
\hline Ovário & 2,63 & 1,81 & 2,62 & 2,01 & 44,57 \\
\hline Oviduto & 3,00 & 4,03 & 2,80 & 3,18 & 34,42 \\
\hline \multicolumn{6}{|l|}{ Comprimento (cm) } \\
\hline Infundíbulo & 8,00 & 7,75 & 6,00 & 7,50 & 20,80 \\
\hline Magno & 37,25 & 36,00 & 34,50 & 37,20 & 11,72 \\
\hline Istmo & 10,75 & 12,25 & 11,75 & 10,50 & 17,54 \\
\hline Glândula da casca & 6,00 & 6,75 & 5,50 & 6,50 & 17,92 \\
\hline Vagina & 4,75 & 3,75 & 2,75 & 2,25 & 37,28 \\
\hline \multicolumn{6}{|l|}{ Número de pregas } \\
\hline Magno & 15,35 & 13,25 & 13,75 & 14,00 & 14,50 \\
\hline Istmo & 8,04 & 12,25 & 13,75 & 12,50 & 13,75 \\
\hline
\end{tabular}

da mucosa. O istmo apresenta luz estreita e mucosa com pregas menores com menor número de glândulas. Tem comprimento de 4 a 12cm, parede muito grossa, com pregas longitudinais e diâmetro reduzido. A glândula da casca ou útero possui parede mais fina que a do istmo, mas apresenta-se fortemente muscular, com pregas longitudinais e transversais e glândulas tubulosas. Tem 4 a 12cm de comprimento, porém é uma região expandida em forma de saco. A vagina possui comprimento de 4 a $12 \mathrm{~cm}$, apresenta pregas longitudinais onde se depositam a maior parte dos espermatozóides após a cópula (MORAES, 2005).

A avaliação macroscópica dos diferentes compartimentos do oviduto está de acordo com a descrição morfológica apresentada anteriormente. Este fato demonstrou que as estruturas estudadas não apresentaram qualquer alteração que designasse presença de alguma patologia.
Em se tratando do peso do ovário, os do presente estudo concordam com os achados de PARK et al. (2004) que, ao trabalharem com diferentes fontes de zinco e com o método de restrição total, não encontraram diferenças para o peso do ovário após a muda entre os tratamentos avaliados. Por outro lado, McCORMICK \& CUNNINGHAM(1987) relataram que a perda de peso do ovário em aves alimentadas com dietas contendo $1 \%$ de zinco durante quatro dias foi de 80\%. BERRY \& BRAKE (1985) demonstraram que a redução do peso do ovário das aves alimentadas com uma dieta contendo $2 \%$ de óxido de zinco, durante oito dias, foi de $30 \%$. A regressão do ovário é o fator mais importante para a indução da muda, pois a sua perda de peso está relacionada com todo o processo de rejuvenescimento. A perda do suporte das gonadotrofinas durante o jejum resulta na regressão do ovário. A redução no peso do ovário depende

Tabela 4 - Peso relativo do ovário e oviduto de poedeiras comerciais submetidas a diferentes tipos de muda forçada.

\begin{tabular}{|c|c|c|c|c|c|}
\hline Dias do experimento & Restrição & Zinco & Cálcio & Sódio & $\mathrm{CV}(\%)$ \\
\hline Peso relativo do ovário & \multicolumn{5}{|c|}{ (\%) } \\
\hline 28 & 0,87 & 1,42 & 1,77 & 1,66 & 61,57 \\
\hline 56 & 1,96 & 1,69 & 1,65 & 1,92 & 20,34 \\
\hline 84 & 1,90 & 1,72 & 1,65 & 1,92 & 22,09 \\
\hline 112 & 2,00 & 1,81 & 1,86 & 2,26 & 27,49 \\
\hline Peso relativo do oviduto & \multicolumn{5}{|c|}{$(\%)$} \\
\hline 28 & 2,33 & 3,74 & 4,11 & 4,30 & 63,80 \\
\hline 56 & 4,71 & 3,53 & 3,09 & 3,61 & 20,64 \\
\hline 84 & 4,32 & 3,75 & 3,09 & 3,61 & 18,81 \\
\hline 112 & 4,34 & 4,63 & 4,36 & 5,91 & 35,83 \\
\hline
\end{tabular}

Ciência Rural, v.37, n.1, jan-fev, 2007. 
Tabela 5 - Comprimento do infundíbulo, magno, istmo, glândula da casca e vagina de poedeiras comerciais submetidas a diferentes tipos de muda forçada.

\begin{tabular}{|c|c|c|c|c|c|}
\hline Dias do experimento & Restrição & Zinco & Cálcio & Sódio & $\mathrm{CV}(\%)$ \\
\hline Infundíbulo & \multicolumn{4}{|c|}{$(\mathrm{cm})$} & \\
\hline 28 & 7,00 & 7,00 & 7,00 & 6,50 & 11,11 \\
\hline 56 & 6,50 & 6,75 & 6,50 & 7,00 & 7,16 \\
\hline 84 & 6,50 & 6,75 & 7,00 & 7,00 & 8,21 \\
\hline 112 & 6,50 & 7,25 & 7,75 & 7,25 & 10,31 \\
\hline Magno & \multicolumn{4}{|c|}{$(\mathrm{cm})$} & \\
\hline 28 & 28,00 & 33,25 & 34,25 & 35,00 & 24,70 \\
\hline 56 & 38,25 & 42,50 & 38,50 & 39,50 & 9,36 \\
\hline 84 & 38,00 & 42,66 & 39,50 & 38,50 & 10,25 \\
\hline 112 & 40,00 & 40,50 & 39,75 & 38,00 & 10,10 \\
\hline Istmo & \multicolumn{4}{|c|}{$(\mathrm{cm})$} & \\
\hline 28 & 7,00 & 9,87 & 9,00 & 9,25 & 30,47 \\
\hline 56 & 13,00 & 11,00 & 12,00 & 11,00 & 16,34 \\
\hline 84 & 13,33 & 11,33 & 12,00 & 11,25 & 17,19 \\
\hline 112 & 8,00 & 11,00 & 10,75 & 9,50 & 22,74 \\
\hline Glândula da casca & \multicolumn{4}{|c|}{$(\mathrm{cm})$} & \\
\hline 28 & 5,50 & 7,50 & 7,75 & 8,50 & 20,80 \\
\hline 56 & 10,00 & 8,25 & 8,75 & 9,25 & 15,02 \\
\hline 84 & 9,66 & 8,66 & 8,75 & 9,25 & 15,12 \\
\hline 112 & 7,50 & 7,50 & 7,75 & 7,00 & 13,85 \\
\hline Vagina & \multicolumn{4}{|c|}{$(\mathrm{cm})$} & \\
\hline 28 & 3,25 & 2,00 & 2,50 & 2,50 & 29,27 \\
\hline 56 & 3,75 & 3,50 & 2,75 & 3,75 & 34,88 \\
\hline 84 & 3,50 & 2,50 & 2,75 & 2,75 & 18,78 \\
\hline 112 & 2,75 & 3,25 & 3,00 & 2,75 & 20,26 \\
\hline
\end{tabular}

inicialmente da duração do jejum e da percentagem do peso corporal. Segundo BERRY (2003), ocorrendo 25\% de perda do peso corporal da ave, o ovário regride completamente.

A regressão do oviduto ocorre após a perda do suporte dos hormônios esteróides do ovário e se dá mais pelo remodelamento deste tecido do que pela diminuição no tamanho das células, ou mesmo por sua contração, quando a apoptose reduz o número de células do epitélio glandular durante esta regressão (HERYANTO et al., 1997).

Tabela 6 - Número de pregas do magno e do istmo de poedeiras comerciais submetidas a diferentes tipos de muda forçada.

\begin{tabular}{|c|c|c|c|c|c|}
\hline Dias do experimento & Restrição & Zinco & Cálcio & Sódio & $\mathrm{CV}(\%)$ \\
\hline Pregas magno & \multicolumn{5}{|c|}{ (nº pregas) } \\
\hline 28 & 15,00 & 15,50 & 14,50 & 15,25 & 5,91 \\
\hline 56 & 15,75 & 15,25 & 15,25 & 14,75 & 7,80 \\
\hline 84 & 15,00 & 15,33 & 15,25 & 14,75 & 6,70 \\
\hline 112 & 14,75 & 15,75 & 15,75 & 13,75 & 7,93 \\
\hline Pregas istmo & \multicolumn{5}{|c|}{ ( $n^{\circ}$ pregas) } \\
\hline 28 & 11,50 & 13,75 & 12,50 & 13,00 & 19,67 \\
\hline 56 & 15,00 & 14,50 & 14,75 & 14,50 & 8,04 \\
\hline 84 & 15,00 & 14,00 & 14,75 & 14,50 & 8,05 \\
\hline 112 & 13,75 & 14,00 & 15,66 & 14,50 & 8,00 \\
\hline
\end{tabular}




\section{CONCLUSÃO}

A indução da muda forçada por intermédio de dietas com alto teor de zinco, baixo nível de cálcio ou sódio mostrou que, para as características macroscópicas do ovário e do oviduto, os resultados obtidos foram similares aos do método convencional de restrição (Califórnia).

\section{AGRADECIMENTOS}

À Fundação de Amparo à Pesquisa do Estado de São Paulo (FAPESP). Processo no. 00/11469-0.

\section{REFERÊNCIAS}

BELL, D.D. Historical and current molting practices in the U.S. table egg industry. Poult Sci, Champaing, v.82, p.965970, 2003.

BERRY, W.D.; BRAKE, J. Comparision of parameters associated with molt induced by fasting, zinc and low dietary sodium in caged layers. Poult Sci, Champaing, v.64, p.20-27, 1985.

BRAKE, J. Recents advances in induced molting. Poult Sci, Champaing, v.72, p.929-931,1993.

BRAKE, J.; THAXTON, P. Physiological changes in caged layers during a forced molt. 1. Body temperature and selected blood constituents. Poult Sci, Champaing, v.58, p.699, 1979a.

BRAKE, J.; THAXTON, P. Physiological changes in caged layers during a forced molt. 2. Gross changes in organism. Poult Sci, Champaing, v.58, p.707, 1979b.

BREEDING, S.W. et al. Molt induced by dietary zinc in a lowcalcium diet. Poult Sci, Champaing, v.71, p.168-180, 1992.

CHRISTMAS, R.B. et al. Performances of single comb white leghorn hens subjected to 4 or 10 day feed withdrawal force rest procedures. Poult Sci, Champaing, v.64, p.2321-2324, 1985.
HERYANTO, B., et al. Involvement of apoptosis and lysosomal hidrolase activity in the oviducal regression during induced molting in chickens: A cytochemical study for end labeling of fragmented DNA and acid phosphatase. Poult Sci, Champaing, v.76, p.67-72, 1997.

KUENZEL, W.J. Neurobiology of molt in avian species. Poult Sci, Champaing, v.82, p.981-991, 2003.

McCORNICKY, C.C.; CUNNINGHAM, D.L. Performance and physiological profiles of high dietary zinc and fasting as methods of inducing a forced rest: a direct comparision. Poult Sci, Champaing, v.66, p.1007-1013, 1987.

MORAES, I.A. Fisiologia da reprodução de aves. 2005. Acesso em: 15 mar. 2005. On line. Disponível em : <www.uff.br/ fisiovet/fisio_rep_aves.htm> online.

NATIONAL RESEARCH COUNCIL. NRC. Nutrientes requeriments of poultry. 9.ed. Washington: National Academic, 1994. 156p.

NORTH, M.O.; BELL, D.D. Commercial chicken production manual. 4.ed. New York: Chapman and Hall, 1990. p.433-452.

PARK, S.Y. et al. Effects of high zinc diets using zinc propionate on molt induction, organs and postmolt egg production and quality in laying hens. Poult Sci, Champaing, v.83, p.24-33, 2004

ROLAND, D.A .; BRAKE, J. Influence of premolt production on posmolt performance with explanation for improvement in egg production due to forced molting. Poult Sci, Champaing, v.61, p.2473, 1982.

SAS. SAS ${ }^{\circledR}$ User's guide: changes and enhancements through release 6.11. Cary, NC, 1996.

SHIPPEE, R.L. et al. High dietary zinc or magnesium as forcedresting agensts for laying hens. Poult Sci, Champaing, v.58, p.949-954, 1979.

WEBSTER, A.B. Phisiology and behavior of the hen during induced molt. Poult Sci, Champaing, v.82, p.992-1002, 2003. 